\title{
Bemerkungen zum „Genetiv“ im Ägyptischen
}

1. In vielen Anfängerlehrbüchern, aber auch in einschlägigen grammatischen Abhandlungen, wird der Terminus „Genetiv“ mit einer gewissen Zurückhaltung eingeführt oder sogar rundheraus als (an sich, eigentlich) ungeeignet für das Ägyptische bezeichnet. So setzt E. Graefe "Genitiv“ in Anführungszeichen, ebenso W. Schenkel, der erläutert, daß die beiden entsprechenden Konstruktionen des Ägyptischen nur ,als funktionale Entsprechungen des Kasus ,Genitiv' anderer Sprachen als ,Genitiv ${ }^{`}$ bezeichnet werden "“. ${ }^{3}$ F. Junge hält die Bezeichnung für gänzlich ungeeignet: „Da das Ägyptische keine Kasus hat, ist "Genitiv' allerdings eine irreführende Bezeichnung ${ }^{{ }^{4}}$. Auch R. Simpson behält den Terminus nur deshalb bei, weil er so fest eingebürgert ist ,in spite of its inappropriate associations with casemorphology in Indo-European languages ${ }^{{ }^{5}{ }^{5}}$, während A. Shisha-Halevy selbst das nicht für richtig hält: „The removal of this ethnocentrism ... from Egyptian linguistic terminology is long overdue, especially in Coptic, where it obscures the identity of the de-

Abkürzungen nach Lexikon der Ägyptologie, Bd. 7, XIII-XXXVIII. Außerdem: EAG = E. Edel, Altägyptische Grammatik, AnOr 34/39, 1955/64; GEG = A. H. Gardiner, Egyptian Grammar, Oxford ${ }^{3} 1957$; LGE = G. Lefebvre, Grammaire des l'égyptien classique, BdE 12, ${ }^{2} 1955$; WGMT $=$ W. Westendorf, Grammatik der medizinischen Texte, Berlin 1962 (Grundriß der Medizin der alten Ägypter, VIII); SpmG = K. Jansen-Winkeln, Spätmittelägyptische Grammatik der Texte der 3. Zwischenzeit, ÄUAT 34, 1996.

Mittelägyptische Grammatik für Anfänger, Wiesbaden ${ }^{5} 1997,21-2$ (\$ 12$)$.

${ }^{3}$ Tübinger Einführung in die klassisch-ägyptische Sprache und Schrift, 1997, 122. In einer älteren Arbeit hatte Schenkel den Verdacht geäußert, „daß der ,Genitiv“ in der Hauptsache aus der Notwendigkeit der Übersetzung in europäische Sprachen sein Dasein fristet" (ZÄS 88, 1962, 58).

${ }^{4}$ Einführung in die Grammatik des Neuägyptischen, Wiesbaden 1996, 61.

Demotic Grammar in the Ptolemaic Sacerdotal Decrees, Oxford 1996, 67. terminative and attributive groups ${ }^{\text {* }^{6}}$. Derartige Stellungnahmen sind auch sonst überaus häufig.

Es ist natürlich richtig, daß es im Ägyptischen keine morphologisch markierten Kasus gibt ${ }^{7}$, und damit hängt es zusammen, daß der ägyptische „Genetiv" nur als Genetivattribut vorkommt, nicht aber in all den Funktionen, die er in Sprachen mit ausgebildeter Kasusmorphologie haben kann, wie etwa als obliques Objekt eines Verbs oder Adjektivs, als adverbialer Genetiv, als Prädikativ („des Todes sein", genetivus pretii), nach bestimmten Präpositionen u. ä. Aber auch in diesen Sprachen ist die Grundund Hauptfunktion des Genetivs immer die der Attribution eines Substantivs („Relativkasus“, „possessive marker" etc.), während die anderen (marginalen) Funktionen in den verschiedenen Sprachen in unterschiedlicher Art und Anzahl vorhanden sind ${ }^{8}$. Die beiden ägyptischen Konstruktionen („,direkter“ und ,indirekter Genetiv“) entsprechen nach Funktion und Bedeutung ziemlich genau dieser Grundund Hauptfunktion des („eigentlichen“, kasusmarkierten) Genetivs, und insofern kann man diese Bezeichnung unter funktionalem Aspekt durchaus guten Gewissens auf sie anwenden?

JEA 66, 1980, 185.

Den status constructus des nomen regens wird man kaum als eine solche Markierung betrachten dürfen, da nicht sicher ist, ob das nomen regens nicht auch in anderen nominalen Fügungen (z. B. vor attributivem Adjektiv) enttont wurde. Zudem und vor allem steht er nicht in Kontrast zu anderen „Kasus“.

${ }^{8}$ In manchen Fällen, wie etwa dem Genetiv nach bestimmten Präpositionen, könnte man sogar zweifeln, ob hier überhaupt von einer Kasusfunktion gesprochen werden kann. Sofern eine Präposition nur einen Kasus regiert (und nicht zwei in unterschiedlicher Bedeutung), ist er eigentlich redundant.

Jedenfalls sofern man sich darüber im klaren ist, daß damit in diesem Fall nur eine syntaktische Relation bezeichnet ist, kein eigentlicher Kasus, vgl. etwa B. J. Blake, Case, Cambridge 1994, 59: „The notion of case is useful only where cases can express more than one relation. There is a danger in the notion of universal, abstract case of confusing case with grammatical relations." 
2. Der gemeinsame Oberbegriff „Genetiv“, unter dem man beide ägyptischen Konstruktionen allgemein zusammenfaßt (gleichgültig, ob mit gutem oder schlechtem Gewissen), geht von einer engen funktionalen Zusammengehörigkeit dieser beiden Konstruktionen aus, aber selbst diese Zusammengehörigkeit ist in Zweifel gezogen worden: W. Schenkel zufolge sind beide Konstruktionen zwar durch einige Gemeinsamkeiten durchaus benachbart, „daraus resultiert jedoch nicht, daß die beiden ,Genitive ' in älterer Zeit enger unter sich als mit anderen Syntagmata verwandt sind ${ }^{\text {“ }}{ }^{10}$. Das ist aber wohl übertrieben skeptisch; man wird zweifellos von einer sehr engen Zusammengehörigkeit von direktem und indirektem Genetiv ausgehen können:

a) Beide Konstruktionen stehen in komplementärer Distribution mit Substantiv + Suffix; wird das Rectum pronominalisiert, gibt es für beide nur diese Möglichkeit (z. B. $\underline{d} 3 \underline{d} 3 t$ (nt) ntr 93 vs. $\underline{d} \underline{d} \underline{d} t . f)^{11}$.

b) Beide Konstruktionen sind in etwa bedeutungsgleich (zu eher marginalen Unterschieden s. u., 〔 7) und können im Satzverband die gleiche syntaktische Position besetzen.

c) Beide Konstruktionen können als freie Varianten voneinander vorkommen (s. u., \& 7, Ende).

d) Bei bestimmten Wörtern stehen die beiden Konstruktionen in komplementärem Verhältnis (s. u., \8).

e) Im Lauf der Sprachgeschichte hat der indirekte Genetiv den direkten bis auf einige lexikalisierte Ausdrücke ersetzt.

Man wird also durchaus davon ausgehen dürfen, daß beide Konstruktionen schon zur Zeit der Pyramidentexte sehr eng zusammengehörig sind und daher zusammen behandelt werden können und müssen (wie es ja Schenkel auch getan hat).

3. In einigen (in älterer Sprache eher marginalen) Bereichen steht der Genetiv in Konkurrenz zu anderen Konstruktionen der nominalen Attribution. Er kann seiner Bedeutung nach öfter einem adjektivischen Attribut entsprechen; so könnte man ein $w \underline{d}$ njswt „Königsbefehl“ auch durch „königlicher Befehl“" wiedergeben oder pr jtj durch ,väterliches Haus". Tatsächliche Parallelbildungen sind allerdings

ZÄS 88, 1962, 60.

Mit anderen Worten: der indirekte Genetiv kann nicht pronominalisiert werden. nicht oft belegt, wie etwa $m w n t r$ "Gotteswasser ${ }^{\text {s }^{12}}$ und $m w$ ntrj ,göttliches Wasser ${ }^{\text {“"13 }}$, beides in der Bedeutung ,göttliche Nachkommen“. Beide Konstruktionen können also nur dann in etwa äquivalent sein, wenn das Rectum in nichtspezifizierender Bedeutung verwendet wird und wenn es ein semantisch entsprechendes Adjektiv gibt (was z. B. bei „königlich“ oder „väterlich“ nicht der Fall ist). Teilweise ist der Gebrauch wohl auch lexikalisch bzw. in der Sprachnorm geregelt: So heißt es grundsätzlich $w 3 t$ ntr „Gottesweg" (und m. W. nie *w3t ntrijt), aber öfter $m r$ ntrj ,göttlicher Teich “14. Ferner gibt es eine besondere "qualifizierende“ Art des indirekten Genetivs, die in ihrer Bedeutung oft einer adjektivischen Attribution entspricht (z. B. z $n \mathrm{~m}^{3} \mathrm{C}_{t}$, ,ein Mann der Wahrheit“ $=$ „ein wahrhaftiger Mann“, entsprechend $z$ m39) und die im Verlauf der Sprachgeschichte tatsächlich allmählich das adjektivische Attribut ersetzt hat, s. u., $\int 9-10^{15}$.

Die Beziehungen zwischen Genetiv und Apposition sind nicht so eng wie die zwischen Genetiv und adjektivischem Attribut. Immerhin gibt es zuweilen alternierenden Gebrauch von Genetiv und Apposition, z. B. $d m j N N$,der Ort NN" und $d m j n N N$ (dto. $)^{16}$. Zudem ist es manchmal zweifelhaft, ob wir es mit einem Genetivattribut oder einer appositiven Relation zu tun haben ${ }^{17}$. Insgesamt aber sind solche Fälle (wenn der Kontext eindeutig ist) äußerst selten ${ }^{18}$.

12 Papyrus Turin 1993, Z. 11 (s. Möller, Hier. Lesestücke, II, 30).

${ }^{13} \mathrm{~Wb} \Pi 1 \mathrm{I}, 363,7$.

${ }^{14} \mathrm{~Wb}$ II, 363, 17.

Allerdings ist es bei dieser Art des ,indirekten Genetivs" sehr zweifelhaft, ob man dabei überhaupt von "Genetiv" sprechen darf, s. u., \& 11.

${ }^{6} \mathrm{~Wb}$ V, 455,15-6. Vgl. für das Demotische auch R. Simpson, Demotic Grammar, 70 (13) (p3 rn [n] Brnyg3 ,the name [of] Berenice $\left.{ }^{6}\right)$ und $72-3$. Es gibt auch im Deutschen gelegentlich Fälle, wo beide Konstruktionen weitgehend bedeutungsgleich sind, z. B. „ein Dutzend frische Eier" (App.) oder „ein Dutzend frischer Eier“ (Gen.).

Vgl. etwa EAG, \327 und 312.

${ }^{8}$ Man beachte auch, daß F. Junge, Einführung in die Grammatik des Neuägyptischen, 63, die Verbindung von kjj ,anderer" (u. Var.) mit seiner lexikalischen Expansion für einen direkten Genetiv hält. M. E. handelt es sich dabei um eine appositive Verbindung, was sich u. a. daran zeigt, daß gelegentlich $k j j$ im status pronominalis (+ Erweiterung) vorkommt, vgl. GEG, $\int 98$ (mit Nachtrag auf p. XXXII). Daß man darüber aber überhaupt streiten kann, zeigt, daß die Abgrenzung beider Konstruktionen nicht immer ganz eindeutig ist. 
Der indirekte Genetiv kann weiter als Ersatz eines exozentrischen Kompositums vom Typ $n f r-h r$ dienen $^{19}$ : statt $z \varsigma$ jqr $d b^{c} w$,ein Schreiber mit fähigen Fingern" kann man auch $z \zeta$ jqr $n \underline{d} b^{\ulcorner} w \cdot f$ sagen, allerdings nur unter ganz bestimmten Voraussetzungen ${ }^{20}$ und insgesamt recht selten. Man beachte, daß die Genetivkonstruktion ein rückbezügliches Pronomen haben muß, während das Kompositum $\left(j q r-d b^{\top} w\right)$ keines haben darf.

4. Nach W. Schenkel steht im Mittelägyptischen der direkte Genetiv ,in mehr oder minder festen Ausdrücken und ist nur in engem Rahmen noch produktiv ..., der indirekte Genitiv ist die gängige Konstruktion "6" Wenn das so ist, müßte man sich fragen, inwieweit der direkte Genetiv überhaupt eine freie Wortverbindung ist oder schon ein Kompositum, anders ausgedrückt, ob er eher in der Syntax oder in der Wortbildung behandelt werden sollte. Nach H. Paul ${ }^{22}$ ist es eine Vorausbedingung für die Klassifizierung einer Wortgruppe als Kompositum, daß 1. „die zugrundeliegende syntaktische Verbindung als Ausdruck eines einheitlichen Begriffes gefasst werden kann, und dies ist nur möglich, wenn wenigstens das bestimmende Element in derselben in seiner allgemeinen Bedeutung $\mathrm{zu}$ nehmen ist und nicht in einer konkreten Individualisierung", und 2. „das Ganze den Elementen gegenüber, aus denen es zusammengefasst ist, in irgend welcher Weise isoliert wird“. Diese Bedingungen sind beim direkten Genetiv - zumindest in älterer Zeit - in sehr vielen Fällen ganz eindeutig nicht gegeben, was schon daran deutlich wird, daß das nomen rectum seinerseits erweitert werden kann, z. B. von einem Suffix, einem weiteren Genetiv, einem Adjektiv oder einer Relativphrase, und damit semantisch qualifiziert und individualisiert wird $^{23}$.

\footnotetext{
${ }^{19}$ Was damit zusammenhängen könnte, daß das Kompositum sekundär als direkter Genetiv reinterpretiert worden ist.

${ }^{20} \mathrm{Vgl}$. ZÄS 121, 1994, 63-5.

${ }^{21}$ Tübinger Einführung 1997, 122.

${ }^{22}$ Prinzipien der Sprachgeschichte, Tübingen ${ }^{1975}$, $\int 228-9$.

${ }^{23}$ Vgl. etwa die Beispiele bei WGMT, \143-5. Natürlich kann auch ein Kompositum erweitert werden, aber dann bezieht sich eine solche Erweiterung auf die gesamte Verbindung: z. B. bedeutet hm-ntr.f ,sein Gottesdiener, sein [des Gottes] Priester", nicht etwa „der Diener seines Gottes“; das Suffix ist auf den Nukleus der Verbindung bezogen. Vgl. allgemein SpmG, \ 127.
}

Eine weitere Bedingung für ein Kompositum ist es, daß seine Bestandteile untrennbar miteinander verbunden sind („Stabilität der Wortstruktur“). Zumindest in ältester Zeit ist auch dies nicht unbedingt gegeben. A. Erman war sogar noch für das Mittelägyptische davon ausgegangen, daß es sich beim direkten Genetiv um eine eher lose Verbindung handele $^{24}$. Beispiele für die Sprengung der angeblichen status constructus-Verbindung sind auch im Mittelägyptischen ${ }^{25}$ wie im Altägyptischen ${ }^{26}$ gar nicht selten. C. E. Sander-Hansen hat auf die erstaunliche Tatsache hingewiesen, daß in den Pyramidentexten in einer ganzen Reihe dieser Fälle das nomen rectum eine Ortsbezeichnung ist. Er hat daraus gefolgert, es läge gar keine Genetivverbindung vor: vielmehr stehe das angebliche nomen rectum in einer Art "casus localis des ältesten Ägyptischen “27. Ein solcher adhoc-Ansatz eines „casus localis" dürfte allerdings wenig wahrscheinlich sein. Zudem bleiben ja immer noch zahlreiche Fälle übrig, wo das Rectum keine Ortsbezeichnung ist.

Insgesamt wird man sagen dürfen, daß der direkte Genetiv des Alt- und Mittelägyptischen kein Kompositum war, sondern eine (freie) Wortfügung. Das schließt natürlich nicht aus, daß die Verbindung in zahlreichen Einzelfällen schon univerbiert worden war und sich $\mathrm{zu}$ einem Kompositum entwickelt hatte $^{28}$. In bestimmten Fällen, den sogenannten „Komposita älterer Bildungsweise ${ }^{c 6^{29}}$ (die z. T. auf Genetivverbindungen zurückgehen), ist das sogar ganz sicher. Ebenso muß sich der direkte Genetiv im Lauf der Sprachgeschichte zu einer ziemlich unproduktiven, nur noch lexikalisiert vorkommenden Bildung im Koptischen entwickelt haben. Welcher Stand der Entwicklung zu einer gegebenen Zeit dazwischen erreicht war, ist allerdings im Einzelfall oft schwer zu beurteilen.

5. Beide Genetivkonstruktionen des Ägyptischen, die direkte und die indirekte, sind schon in den ältesten Texten reichlich bezeugt. Dennoch wird man sa-

${ }^{24} \ddot{\mathrm{AG}}{ }^{4} 1928, 』 214$.

${ }^{25}$ Vgl. WGMT, \& 146 (unter bb) und LGE, \ 150.

${ }^{26}$ Vgl. EAG (\$ 321: durch attributive Elemente; \ 322: durch die Kopula pw; § 323: durch $j$, ,wie (").

${ }^{27}$ AcOr 14, 1936, 27-8.

${ }^{28}$ Zur Erkennbarkeit von Komposita im Ägyptischen vgl. generell SpmG, \ 125-9.

29) Vgl. dazu Fecht, Wortakzent, passim sowie Schenkel, Einführung in die altägyptische Sprachwissenschaft, Darmstadt 1990, $81 \mathrm{ff}$. 
gen dürfen, daß die indirekte, analytische Verbindung typologisch jünger ist, und es ist ja auch die Form, die den direkten Genetiv allmählich verdrängt hat.

Beide Konstruktionen sind teils komplementär, teils konkurrierend, teils freie Varianten (s. u.), und das erschwert das Erkennen eventueller Bedeutungsunterschiede zwischen ihnen zusätzlich. Ob es solche Bedeutungsunterschiede gibt und worin sie bestehen, ist unterschiedlich beantwortet worden. A. Erman hatte in seiner Grammatik $^{30}$ nur einige charakteristische Vorkommensweisen des direkten Genetivs (\$ 216: nach allgemeinen Ortsausdrücken, z. B. st, nach Wörtern für „Haus“ oder „Herr, Vorsteher, König“) und des indirekten Genetivs ( $(218 / 218$ a: das nomen rectum bezeichnet den Besitzer, den Stoff, den Teil, oder es hat fast den Wert eines adjektivischen Attributs) aufgeführt. In den Grammatiken von G. Lefebvre ${ }^{31}$ und E. Edel ${ }^{32}$ werden die unterschiedlichen syntaktischen Verhaltensweisen beider Konstruktionen, ihre teilweise Austauschbarkeit und ihre jeweiligen speziellen Verwendungen aufgeführt, ohne einen Generalnenner für die Unterschiede zu suchen.

Solche generellen Unterschiede sind aber verschiedentlich vorgeschlagen worden: C. E. SanderHansen $^{33}$ sah den Unterschied in der morphologischen Struktur des nomen regens: hat es eine unbetonte Endung (wie oft im Plural und Dual, im Feminin, bei Nomina auf. $w$ und .wt sowie bei Nisben), zieht es den direkten Genetiv nach sich, ist die Endsilbe betont, folgt der indirekte Genetiv (wobei Sander-Hansen für den ersten Fall nur mit wenigen Ausnahmen rechnete, während beim zweiten, indirekter Genetiv nach betonter Endsilbe, die Ausnahmen "außerordentlich zahlreich" sein sollten ${ }^{34}$ ). H. Junker ${ }^{35}$ hielt den direkten Genetiv dann für zwingend, wenn das Regens das Rectum in Besitz hat, während bei Abhängigkeit des Regens vom Rectum beide Konstruktionen möglich sind.

In dem Anfängerlehrbuch von P. Grandet und B. Mathieu ${ }^{36}$ wird als Regel gegeben, daß im indirek-

${ }^{30}$ ÄG, ${ }^{4} 1928$.

${ }^{31}$ LGE, \ $140-53$.

${ }^{32} \mathrm{EAG}, § 318-31$.

3 ,Zum Gebrauch des direkten und indirekten Genitivs in der ältesten ägyptischen Sprache", AcOr 14, 1936, 26-54.

${ }^{34}$ Ibid., 33 .

${ }^{35}$ Gîza III, 94.

${ }^{36}$ Cours d'égyptien hiéroglyphique, I, Paris 1990, 89-94. ten Genetiv das nomen rectum definit ist (bzw. durch diese Konstruktion selbst determiniert wird: $n h t ~ n(t) n b$,le sycomore du maitre"), im direkten Genetiv dagegen indefinit ( $n h t n b$,le sycomore d'un maitre"). Allerdings kann im direkten Genetiv das nomen rectum per se definit sein (bei Namen, Abstrakta, Nomen + Suffix, absolut-realen Begriffen etc.). Mithin würde die Regel letztlich lauten: in all jenen Verbindungen, wo das nomen rectum aufgrund der lexikalischen Besetzung unbestimmt sein kann, ist es im direkten Genetiv unbestimmt, im indirekten Genetiv bestimmt.

F. Junge gibt in seiner Einführung ins Neuägyptische $^{37}$ eine fast genau entgegengesetzte Erklärung: Der direkte Genetiv sei restringierend, der indirekte qualifizierend (und zwar v. a. in älterer Sprache, im Neuägyptischen selbst dann nicht mehr bzw. kaum noch). Die beiden letztgenannten Definitionsversuche dürften schon auf den ersten Blick große Schwächen haben (ganz abgesehen davon, daß sie sich widersprechen): Es ist nicht einzusehen, warum Jmn in $\mathrm{pr} J \mathrm{mn}$ mit Junge restringierend sein sollte und $J n p w$ in hwt-ntr $n t$ Jnpw qualifizierend, entsprechend qsnt in hrw qsnt „Tag des Unheils ${ }^{4{ }^{38}}$ restringierend, $m n j$ in hrw $n$ mnj "Tag des Sterbens ${ }^{639}$ qualifizierend. Ein nomen rectum von $j t j$,Vater" und $m w t$,Mutter" (des NN) sollte in der Regel restringierend sein; tatsächlich folgt aber sehr oft der indirekte Genetiv ${ }^{40}$. Und die zahlreichen Varianten ${ }^{41}$ sprechen ebenso dagegen wie das unten $\left(\int 8\right)$ geschilderte Komplementaritätsverhältnis bei bestimmten Wörtern. Der Unterschied zwischen direktem und indirektem Genetiv kann mit restringierend vs. qualifizierend nichts zu tun haben. Konsequenterweise ist er ebensowenig davon abhängig, ob das nomen rectum bestimmt oder unbestimmt ist. Hätten Grandet/Mathieu recht, würde das ja bedeuten, daß Verbindungen wie $m r m s^{r}$ „Armeebefehlshaber“, $h q 3 \quad h 3 s w t$ „Herrscher der Fremdländer“, $n b t$ pr „Hausherrin“ etc. grundsätzlich indeterminiert wären, denn in einer Genetivverbindung wird der Grad der Determinierung ausschließlich vom nomen rectum bestimmt ${ }^{42}$. Man

\footnotetext{
61.

${ }^{37}$ Einführung in die Grammatik des Neuägyptischen, ${ }^{38}$ Lebensmüder, 15.

39 Erman, $\ddot{A} G, § 218$ a.

${ }^{40}$ Vgl. Sander-Hansen, AcOr 14,36; 41.

${ }^{41}$ Vgl. etwa LGE, $\int 146: h r w(n) z m 3-t 3$ sowie die Angaben unten, \& 7 , Ende.

${ }^{42}$ Vgl. H. Weinrich, ,Textsyntax des französischen Artikels“ in: Sprache in Texten, Stuttgart 1976, 197-8.
} 
vergleiche auch die Titel (bzw. Funktionsbezeichnungen) $m r n w t$ "Stadtvorsteher" (immer direkter Genetiv $^{43}$ ) vs. h3tj-c $n n w t$ „Bürgermeister“ (immer indirekter Genetiv ${ }^{44}$ ); ein Unterschied im Grad der Definitheit ist nicht festzustellen.

Das grundsätzliche Problem bei H. Junkers Abgrenzungsversuch ist, daß darin die außersprachlichen (also referentiellen) Abhängigkeitsverhältnisse die Art der Genetivverbindung bestimmen sollen. Ein „Herr" als regens z. B. würde den direkten Genetiv erfordern, ein „Diener“ dagegen könnte beide Genetivarten nach sich ziehen. Gegenbeispiele, die Junkers Ansatz widerlegen, hat Edel gegeben ${ }^{45}$. Zudem finden sich sehr viele Fälle, wo von einem solchen (außersprachlichen) Abhängigkeitsverhältnis ohnehin nicht gesprochen werden kann (z. B. dpt $m w t$ „Geschmack des Todes“, st sfwt „Stelle der Geschwulst" etc.).

Bei Sander-Hansen hat man zunächst einmal das (eingestandene) Problem, daß seine These von vornherein mit sehr vielen Ausnahmen belastet ist. $\mathrm{Zu}$ dem kann man damit die nicht seltenen Varianten nicht erklären, wenn hinter ein und demselben Wort bald der direkte, bald der indirekte Genetiv vorkommt ${ }^{46}$. Man wird allenfalls zugestehen, daß die Struktur des nomen regens die Wahl der Genetivverbindung beeinflussen kann (und das zumindest in einigen Fällen auch ganz regelmäßig tut, s. u., \8).

Generell lassen alle diese Erklärungsversuche die zahlreichen Varianten (s. u., §7, Ende) unbeachtet, die ja nicht alle fehlerhaft sein können.

Eine Erklärung, die schwieriger zu widerlegen ist, hat W. Schenkel gegeben ${ }^{47}$. Ihm zufolge ist beim direkten Genetiv die Zugehörigkeit des Rectum zum Regens enger als beim indirekten Genetiv. Diese enge Zugehörigkeit kann von der lexikalischen Besetzung des Regens beeinflußt werden: So bezeichne $m r j$ „lieben“ ein engeres Verhältnis als $h z j$ „loben“ und tendiere daher (in nominalen Ausdrücken) zum direkten Genetiv. Aber je nach Kontext und Einzelfall kann auch bei gleichem Regens der (engere) direkte Genetiv oder der (weitere) indirekte stehen. In der Tat sollte man ja von vornherein erwarten,

${ }^{43} \mathrm{~Wb}$ II, 212, 2.

${ }^{44} \mathrm{~Wb}$ II, 212,3.

${ }^{45}$ EAG, \ 319.

${ }^{46}$ Vgl. etwa EAG, § $318-9$.

47 "Direkter und indirekter ,Genetiv" ", ZÄS 88, 1962 , $58-66$. daß eine (ursprünglich) periphrastische Form wie der indirekte Genetiv, eine lose, leicht trennbare syntaktische Fügung, auch die semantisch weitere Verbindung ist, dagegen eine unmittelbare Fügung wie der direkte Genetiv, die nur sehr eingeschränkt trennbar ist, auch die semantisch engere. Der Gedanke der engen und weiten Verbindung ist daher naheliegend und findet sich auch bei Gardiner ${ }^{48}$ oder SanderHansen $^{49}$ (bei dem er sozusagen als eine Art untergeordnetes Prinzip erscheint) ${ }^{50}$. Der Vorteil dieses Ansatzes ist seine relative Vagheit: Man kann sich die Zugehörigkeit von Regens und Rectum leicht etwas enger oder weiter vorstellen. Und in dem Fall, wo es offenkundig freie Varianten zwischen direktem und indirektem Genetiv gibt, wäre der Unterschied eben neutralisiert bzw. so geringfügig, daß beide Konstruktionen akzeptabel waren. Aber dies ist natürlich zugleich der Nachteil einer solchen Erklärung: Was überall in etwa passend ist, erklärt nicht viel ${ }^{51}$. Und dennoch wird auch diese recht vage Erklärung immer noch nicht diejenigen Fälle erfassen, wo eine Art komplementäres Verhältnis besteht: bei singularischem Regens indirekter Genetiv, bei pluralischem direkter (s. u., $\left.\int 8\right)^{52}$.

Alle bisher besprochenen Erklärungen versuchen, die Wahl der jeweiligen Genetivkonstruktion auf ein einziges Prinzip zurückzuführen. Aber das ist offenbar nicht möglich; man muß sich also überlegen, ob nicht - insgesamt - verschiedene Ursachen in Frage kommen könnten.

6. Am offenkundigsten sind die syntaktischen Unterschiede zwischen direktem und indirektem Genetiv. Als syntaktische Alternative des direkten Genetivs (sozusagen als periphrastische Form) dient der indirekte Genetiv meist dann, wenn nomen regens $\left(\mathrm{N}_{1}\right)$ und rectum $\left(\mathrm{N}_{2}\right)$ nicht unmittelbar aufeinanderfolgen. Trennung von $\mathrm{N}_{1}$ und $\mathrm{N}_{2}$ im indirekten Genetiv ist belegt:

${ }^{48}$ GEG, \ 85.

49 AcOr 14, 1936, 54.

${ }^{50}$ Man vergleiche für das späte Mittelägyptisch auch SpmG, \4 402 .

${ }^{51}$ Und für Übersetzungen ins Ägyptische (etwa bei der Rekonstruktion von Lücken) wäre sie kaum brauchbar; das zeigt, wie wenig konkret sie ist.

${ }^{52}$ Bemerkenswert ist auch, daß diese Komplementarität gerade bei den Körperteilbezeichnungen sehr häufig ist, wo man (im Singular nicht weniger als im Plural) eine sehr enge Zugehörigkeit annehmen sollte. 
a) durch ein adjektivisches bzw. pronominales (inkl. $n b)$ Attribut

b) durch die Kopula $p w^{54}$;

c) durch ein Adverb oder eine Präpositionalphrase ${ }^{55}$;

d) durch eine Partikel ${ }^{56}$;

e) wenn $\mathrm{N}_{1}$ ein Suffixpronomen hat ${ }^{57}$;

f) durch $r^{\prime}-p w$, ,oder" und $h m w t-r^{\prime}$, ,etc., ${ }^{, 58}$;

g) durch eine Apposition zu N ${ }_{1}^{59}$;

h) wenn es mehrere Regentia gibt, wie es in den Anrufen an die Lebenden nicht selten vorkommt ${ }^{60}$.

Allerdings bedeutet das nicht, daß in diesen Fällen der indirekte Genetiv obligatorisch den direkten ersetzt. Es gibt durchaus Alternativen zu diesen Konstruktionen, mit dem direkten Genetiv oder auf andere Art:

$\mathrm{Zu}$ a) Die Stellung $\mathrm{N}_{1}-$ Attribut $-\mathrm{N}_{2}$ ist auch für den direkten Genetiv in älterer Zeit gelegentlich bezeugt (s. o., \4), allerdings auch dort sehr viel seltener als die entsprechende indirekte Konstruktion. Eine Alternative dazu ist $\mathrm{N}_{1}-\mathrm{N}_{2}$ (im direkten Genetiv) + Attribut ${ }^{61}$.

$\mathrm{Zub}$ ) Hier ist die (nicht seltene) Alternative im direkten Genetiv $\mathrm{N}_{1}-\mathrm{N}_{2}-p w$ bzw. - wenn $\mathrm{N}_{2}$ mehrteilig ist $-\mathrm{N}_{1}-p w-\mathrm{N}_{2}$, s. o., $\int 4$.

Zu c) In diesem Fall kann die Adverbialphrase natürlich ebensogut hinter dem Rectum eines direkten Genetivs stehen.

Zu d) Auch hier wäre die Alternative die Stellung der Partikel hinter der gesamten Genetivverbindung $^{62}$.

Zu f) $r^{\prime}-p w$ kann auch hinter einer direkten Genetivverbindung stehen ${ }^{63}$.

$\mathrm{Zu} \mathrm{g)} \mathrm{Auch} \mathrm{eine} \mathrm{Apposition} \mathrm{kann} \mathrm{natürlich} \mathrm{auf} \mathrm{eine}$ direkte Genetivverbindung folgen (überaus häufig z. B. in Hymnen).

${ }^{53}$ Vgl. etwa EAG, \326; LGE, \$ 149-50; WGMT, $\int 146$; für das spätere Mittelägyptisch SpmG, $\int 400$.

${ }^{54}$ Vgl. LGE, \ 152.

55 Vgl. GEG, \ 121; LGE, \ 151; WGMT, \ 146.

${ }^{56}$ Z. B. PT 801 c: $w r$ js $n$ Jwnw.

${ }^{57}$ Vgl. LGE, $\int 148$ [3 Belege]; GEG, p. 138 [b3k.f $n$ $\underline{d} t . f]$; häufig im späten Mittelägyptisch, vgl. SpmG, $\int 400$.

Vgl. WGMT, § 146.

${ }^{59}$ Selten, vgl. WGMT, \146; ein sicherer Beleg aus späterer Zeit in SpmG, \400 (1. Bsp.: hm-ntr Hr n r'-pr pn „Prophet Hor von diesem Tempel").

${ }^{60}$ Z. B. Sethe, Lesestücke, 87,21-22: hmmw-nțr ḥmwt$n t r w^{\top} b w$ hnw hnwt nw r'-pr pn.

${ }^{61}$ Vgl. etwa EAG, \ 321; GEG, \ 85.

${ }^{62}$ Vgl. (für eine andere Partikel) EAG, \ 323.

${ }^{63}$ Vgl. etwa pEbers, 71,6.
Zu h) Für diesen Fall sind mir keine konkreten Alternativbelege bekannt. Theoretisch gäbe es mehrere Möglichkeiten, z. B. direkter Genetiv und Anschluß der weiteren "regentia" durch

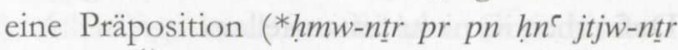
$\left.w^{5} b w \ldots\right)^{64}$ oder den Ersatz des rectum durch eine adjektivierte Präpositionalphrase $\left({ }^{*} h m w\right.$ ntr jtjw-ntr w'bw ntjw m pr pn).

Nur bei e) bietet sich auf den ersten Blick nicht so leicht eine Alternative, weil hier der indirekte Genetiv fast adjektivische Bedeutung hat. Man müßte im Einzelfall $\mathrm{zu}$ unterschiedlichen Umschreibungen (attributive Adjektive, Relativsätze etc.) greifen.

Wenn also der indirekte Genetiv kaum jemals eine unbedingt notwendige, unabdingbare Konstruktion ist, eine Verbindung, deren Bedeutung man nicht auch mit anderen Mitteln ausdrücken könnte, so erlaubt er doch oft einfachere und syntaktisch „losere" Fügungen als der direkte; und daraus ergeben sich eine freiere Wortstellung und ein flexiblerer Satzbau. Er ist ein Mittel, das die syntaktischen Möglichkeiten des Ägyptischen beträchtlich erweitert, und das dürfte in vielen Fällen der Grund für seine Verwendung gewesen sein, ohne daß notwendigerweise ein spürbarer Bedeutungsunterschied zu den Alternativkonstruktionen vorhanden sein müßte.

7. $\mathrm{Ob}$ und inwieweit semantische Unterschiede zwischen beiden Genetivkonstruktionen bestehen (können), ist schon schwieriger zu sagen. Zunächst einmal ist festzuhalten, was mit ,semantischem Unterschied" gemeint ist und was nicht.

Nicht gemeint ist die im Einzelfall spezifische semantische Relation zwischen Regens und Rectum, wie etwa beim genetivus subiectivus, obiectivus, qualitatis, possessivus, auctoris, explicativus etc. Solche besonderen Relationen sind zwar auch für das Ägyptische aufgestellt worden ${ }^{65}$, aber diese besonderen Bedeutungen sind nicht der Genetivrelation als solcher inhärent ${ }^{66}$, sondern sie ergeben sich aus Genetivrelation + lexikalischer Bedeutung von Regens und/oder Rectum, bzw. aus Genetivrelation + lexi-

\footnotetext{
${ }^{64}$ Allerdings schließt $h m$-ntr üblicherweise sein Rectum nur im indirekten Genetiv an.

${ }^{65}$ Vgl. etwa WGMT, $\int 143$; H. von Deines/ W. Westendorf, Wörterbuch der medizinischen Texte, I, Berlin 1961, 425ff. [für den indirekten Genetiv]; Simpson, Demotic Grammar, 69-70.

${ }^{66}$ Vgl. Simpson, op. cit., 70-1; G. Goldenberg, „Attribution in Semitic Languages", Langues orientales anciennes, philologie et linguistique 5-6, 1995, 2 -6.
} 
kalischer Bedeutung + Kontext. Für die Bedeutung der (grammatischen) Genetivrelation als solcher sind diese Einteilungen irrelevant ${ }^{67}$.

Die Genetivverbindung $\mathrm{N}_{1}(n) \mathrm{N}_{2}$ hat üblicherweise (s. u., $\int 10$ ) die allgemeine Bedeutung $\mathrm{N}_{1}$ ist zugehörig $\mathrm{N}_{2}^{68}$. Bei der Frage nach einem semantischen Unterschied zwischen dem direkten und dem indirekten Genetiv geht es zunächst darum, ob der direkte Genetiv eine engere Zugehörigkeit ausdrückt als der indirekte, wie das ja von W. Schenkel und anderen behauptet worden ist (s. o., $\int 5$ ). Wenn mir auch Schenkels Demonstration der unterschiedlichen Konstruktionen des Lexempaares $m r j$ und $h z j$ für eine so allgemeine Schlußfolgerung nicht ganz ausreichend erscheint, so kann man immerhin noch auf einige andere Punkte verweisen, die in die gleiche Richtung deuten:

a) $\mathrm{Da}$ eine Verbindung durch unmittelbare Nebeneinanderstellung (dazu noch in der Regel untrennbar und wenigstens z. T. mit Druckverlust des 1. Glieds) eine engere Beziehung signalisieren könnte als eine lockere periphrastische Konstruktion, scheint a priori naheliegend.

b) Der direkte Genetiv ist besonders geläufig in Titeln und titelähnlichen Verbindungen, in Filiationsangaben und anderen festen Phrasen.

c) Offenbar drückt man einheitliche Begriffe (wie sie etwa den deutschen Determinativkomposita entsprechen) lieber im direkten Genetiv aus, z. B. $n b$ pr „Hausherr”, nb jht „Eigentümer, Besitzer,"69, aber $n b n$ jšt.f $n b$ „Herr all seines Besitzes" ${ }^{\text {, }}$. Das dürfte doch wohl damit zusammenhängen, daß der direkte Genetiv formal kompakter ist als der an beiden Gliedern beliebig erweiterbare indirekte und sich dadurch besser als Form eines einheitlichen Begriffes eignet (und auch eher zur Univerbierung neigte).

Insgesamt betrachtet wird aber dieser semantische Unterschied zwischen direktem und indirektem Ge-

\footnotetext{
${ }^{67}$ Obwohl derartige Angaben in Lehrbüchern durchaus nützlich sein mögen, insofern man für die Übersetzungspraxis auf häufige Verbindungen hingewiesen wird, auf besondere Bedeutungen oder alternative Verständnismöglichkeiten (wie bei amor matris).

${ }^{68}$ Wobei ,zugehörig" rein sprachlich gemeint ist: „der Herr des Sklaven“ und ,der Sklave seines Herrn“ drücken beide dieselbe sprachliche Zugehörigkeit oder Zuordnung von Regens zu Rectum aus, unabhängig von den außersprachlichen Besitzverhältnissen.

${ }^{69} \mathrm{~Wb}$ II, 228, 5 .

${ }^{70} \mathrm{~Wb}$ II, 228,9; vgl. GEG, p. 423.
}

netiv allenfalls tendenziell vorhanden sein. Daneben gibt es zahlreiche Belege dafür, daß direkter und indirekter Genetiv mehr oder weniger freie Varianten sein können, ohne jeden spürbaren Bedeutungs- oder Funktionsunterschied, vgl. die Beispiele in den Grammatiken von Edel $^{71}$ und Westendorf ${ }^{72}$. Entsprechende Belege sind viel zu zahlreich, als daß man sie als Versehen abtun könnte ${ }^{73}$. Allerdings muß man bei unserer unvollkommenen Kenntnis des Ägyptischen natürlich immer damit rechnen, daß es Nuancen gibt, die wir nicht wahrnehmen.

8. Neben syntaktischen und semantischen Gründen kann noch etwas anderes für die Wahl des direkten oder indirekten Genetivs wichtig sein, nämlich die lexikalische Besetzung und Wortform des nomen regens, denn offenbar haben bestimmte Wörter und Wortformen die Eigenschaft, als nomen regens einen der beiden Genetive zu implizieren. Das ist ganz offensichtlich bei den einsilbigen Maskulina (v. a. Namen von Körperteilen wie $j b, r, t p, h r,{ }^{c} w, r d$, aber auch $k 3, r n$ u. a. m.), die in den Pyramidentexten im Singular das Rectum im indirekten Genetiv anschlieBen, im Dual und Plural aber im direkten ${ }^{74}$. Diese Praxis ist auch keineswegs auf die Pyramidentexte beschränkt (vgl. etwa $h m n$,Majestät des ${ }^{c^{475}}$ ), Spuren dieser Verteilung sind noch im Spätmittelägyptischen zu finden ${ }^{76}$. Aber auch sonst scheint das nomen regens oft Präferenzen für eine bestimmte Genetivkonstruktion zu haben ${ }^{77}$. Diese Regeln im einzelnen (und für die verschiedenen Epochen) zu entschlüsseln, würde umfangreiche Statistiken erfordern. Immerhin läßt sich soviel sagen, daß auch Lexem und Form des nomen regens die Auswahl des Genetivs bestimmen können.

Wie das zu erklären ist, stehe dahin. Der oben (§5) erwähnte Vorschlag von Sander-Hansen ist weitgehend auf Ablehnung gestoßen und in dieser Form wohl auch nicht haltbar. Es dürfte dennoch sehr wahrscheinlich sein, daß diese Komplementarität der Einsilber (indirekter Genetiv im Singular,

${ }^{71}$ EAG, $₫ 318(h t[n t] ~ r m t n b ; d 3 d 3 t$ [nt] ntr (3); \319 (mr $[n] w d t-m d w$ nbt $n t \quad n j s w t ; m r[n] t 3 \quad r-d r . f ;$ shd $[n]$ $h m w-k 3)$.

72 WGMT, § $142-3$.

${ }_{73}^{73}$ Für das späte Mittelägyptisch vgl. auch SpmG, \403.

${ }^{74}$ Vgl. Sander-Hansen, AcOr 14, 1936, 33 ff.; EAG, $\int 324$.

${ }^{75} \mathrm{~Wb}$ III, 91 .

${ }^{76}$ Vgl. SpmG, p. 246 (mit n. 1).

77 Vgl. Sander-Hansen, op. cit., 33 ff.; SpmG, \ 401. 
direkter im Dual und Plural) eher morphologisch lautliche Gründe hat als semantisch - funktionale ${ }^{78}$.

9. Mögliche semantische Unterschiede zwischen direktem und indirektem Genetiv sind oben in $\int 7$ erörtert worden. Es gibt nun noch einen weiteren semantischen Unterschied, aber dieser Unterschied hat nichts mit enger oder weiter Verbindung zwischen Regens und Rectum zu tun, und er ist zudem in der Literatur v. a. für den indirekten Genetiv geltend gemacht worden.

A. Erman unterscheidet für das Neuägyptische zwei Fälle im Gebrauch des indirekten Genetivs: „In dem ersteren bezeichnet er den Besitz oder die $\mathrm{Zu}$ gehörigkeit; das zweite Wort hat dabei den Artikel oder ist sonst bestimmt ${ }^{4{ }^{79}}$. „Der zweite Fall des indirekten Genetivs ist der, wo dieser einen erläuternden Zusatz beifügt. Das zweite Wort bleibt dabei ohne Artikel, da es ja nicht ein einzelnes Exemplar bezeichnet. Wir geben diese Genetive [der zweiten Art] oft durch ein Adjektiv oder ein zusammengesetztes Wort wieder ${ }^{480}$. Als Beispiele für Fall 1 gibt Erman einige Belege für die Bezeichnung des Besitzes und die Zugehörigkeit zu einem Ort oder Amt, für Fall 2 Beispiele für die Angabe des Stoffes ( $\underline{w} w n h m t j$,,ein Berg aus Bronze"), von Inhalt und $\mathrm{Maß}$ ( $w^{r} n t b n$ hnqt „ein Krug Bier“, p3 m3m3 3 n 60 mh „die große Palme von 60 Ellen"), Art oder Herkunft ( $q s$ n rmtw „Menschenknochen“, bj3 $n$ pt „Himmelserz"), allgemein den Bezug (sb3yt $n$ st , ,briefliche Lehre") oder eine abstrakte Qualität ( $p 3 j . j{ }^{`} n h n m^{\top} t$, mein wahrhaftiger Eid").

Die heute allgemeine Ansicht zu diesem Unterschied hat J. Osing folgendermaßen zusammengefaßt: „Beim indirekten Genetiv sind im Neuägyptischen und im Demotischen zwei Gebrauchsweisen deutlich geschieden:

1. Ausdruck der Zugehörigkeit oder Abhängigkeit des Regens im Verhältnis zum Rectum, und

\footnotetext{
${ }^{78}$ Man könnte z. B. als Grund vermuten, daß bei einem Einsilber (mit notwendigerweise kurzem Tonvokal im Singular) eine quantitative Reduktion (von Lang- zu Kurzvokal) im status constructus nicht möglich war, wohl aber im Plural oder im Feminin (und auch im Dual wäre eine lautliche Reduktion wohl einfacher als bei einem Einsilber).

${ }^{79} \mathrm{NG}, \int 208$.

${ }^{80}$ Ibid., $\$ 209$
}

2. qualifizierende Bestimmung des Regens durch das Rectum (Angabe von $\mathrm{Ma} ß$ und Zahl, Stoff, Inhalt, Art und Weise u. ä.)",

W. Spiegelberg hat für das Demotische diese beiden Gebrauchsweisen schon auf zwei unterschiedlich benannte Konstruktionen verteilt, den ,indirekten Genetiv mit $n^{\text {“82 }}$ und das „Adjektivum “83 Trennung wird auch (grundsätzlich, nicht terminologisch) in neuesten Arbeiten zum Demotischen beibehalten $^{84}$. Im Koptischen schließlich werden beide Konstruktionen immer getrennt behandelt (auch wenn die althergebrachten Bezeichnungen als "Genetiv" vs. „Attribut ${ }^{485}$ öfter kritisiert worden sind).

Diesen Unterschied innerhalb des indirekten Genetivs gibt es aber nicht erst seit dem Neuägyptischen. Wenn er in den Grammatiken des älteren Ägyptisch nicht expressis verbis erwähnt wird, liegt das zweifellos daran, daß erst die neuere Sprachstufe den Determinationsgrad formal kennzeichnet und damit eine klare Unterscheidung beider Konstruktionen ermöglicht (ansatzweise im Neuägyptischen, deutlicher im Demotischen, ganz eindeutig erst im Koptischen, weil erst dort das Verhältnis unbestimmter Artikel vs. artikellos indeterminiert systematisiert worden ist). Dennoch ist er auch schon in älterer Zeit in der Sprache (wenn auch nicht in gleichem Umfang) vorhanden. Das läßt sich deutlich daran erkennen, daß bestimmte lexikalische Besetzungen des nomen rectum auch in alter Sprache immer den indirekten Genetiv erfordern. Im Altägyptischen gilt das für Stoffangaben (z. B. 3twt $n t$ $h b n j$,ein Bett aus Ebenholz $)^{86}$, Längenangaben (z. B. jz n $100 \mathrm{mh} m$ 3w.f, „ein Grab von 100 Ellen Länge $\left.{ }^{67}\right)^{87}$ und Inhaltsangaben (hnw nw mnht ,Truhen [voll] Kleidung"; pht nt 2600 „Garbenhaufen von $2600\left[\text { Stück] }{ }^{6}\right)^{88}$. Für das Mittelägyptische bemerkt

${ }^{81}$ J. Osing, Der spätägyptische Papyrus BM 10808, ÄA 33, 1976, 22.

${ }^{82}$ Demotische Grammatik, $₫ 62-3$ (wobei einiges aus $\int 63$ offensichtlich nicht dorthin gehört).

${ }^{83}$ Ibid., $\int 72-3$.

${ }^{84}$ Vgl. etwa Simpson, Demotic Grammar, 67-73 (vgl. auch 80-2); 51-2.

${ }_{85}$ Vgl. W. Till, Koptische Grammatik, Leipzig ${ }^{2} 1961$, \111-3; 114-22; weitere Angaben bei A. ShishaHalevy, Coptic Grammatical Chrestomathy, OLA 30, 1988,$6 ; 8$.




Erman, daß der indirekte Genetiv bei der Angabe des Stoffes (mhrw $n w \underline{d}{ }^{c} m$,Milchkrüge aus Gold $\left.{ }^{\circ}\right)$ und des Teils (nhj $n$ ' gwt ,etwas Schrot $\left.{ }^{\circ}\right)$ gebraucht wird ${ }^{89}$ sowie sehr häufig ,um einem Substantiv einen erläuternden Zusatz beizufügen, oft $\mathrm{da}$, wo wir ein Adjektiv oder einen Relativsatz verwenden würden" (z. B. z $n m^{\circ} t$, „ein Mann der Wahrheit" = „ein wahrhaftiger Mann") ${ }^{90}$. Eine ähnliche Darstellung findet sich in Lefebvres Grammatik: Auch er verweist auf den Gebrauch des indirekten Genetivs ,pour introduire une notion de quantité (années, mesure, multitude, etc) " ${ }^{91}$ und behandelt besonders ausführlich ,le génetif indirect dans certaines expressions où il remplace un adjectif“ (z. B. qmyt nt stj ,duftendes Harz" oder $m s$.f $n$ nhtw ,seine siegreiche Armee" $)^{92}$.

All dies sind Gebrauchsweisen des indirekten Genetivs, die 1. der Konstruktion entsprechen, die sich im späteren Ägyptisch dann zum (von $\mathbf{N}$ eingeleiteten) „Attribut" entwickelt hat und 2. immer nur im indirekten Genetiv vorkommen, so gut wie nie im direkten ${ }^{93}$. Wenn diese besondere Bedeutung des indirekten Genetivs in älterer Zeit auch insgesamt noch seltener sein wird als im jüngeren Ägyptisch, ist sie doch gut belegt. Auf welche Weise sie sich sprachlich von den anderen Verwendungsweisen des indirekten Genetivs abhebt, bedarf also einer Erklärung.

10. Der Unterschied zwischen beiden Konstruktionen liegt zunächst darin, daß das nomen rectum im einen Fall spezifische Bedeutung hat, eine konkrete, identifizierbare Entiät beschreibt, im anderen dagegen aus einem Gattungsbegriff (einem Appellativum), einem Abstraktum oder einer Quantitätsangabe (u. ä.) besteht. Ist das Rectum eine identifizierbare Entität, haben wir es also mit einem „restringierenden" Genetiv zu tun: die referentielle Identität der Gesamtverbindung wird durch das Rectum klargestellt. Im anderen Fall könnte man dagegen von einem "qualifizierenden" Genetiv sprechen: das

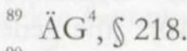

Ibid., \ $218 \mathrm{a}$.

LGE, $\int 147$.

Ibid., \148.

${ }^{3}$ Die einzige Ausnahme wäre allenfalls $h d p^{c} r$,Naos (oder Keule?) aus Nadelholz“ (PT 185a, vgl. EAG, \$327). Eine Apposition ist vielleicht nicht ausgeschlossen (vgl. EAG, \ 312). Auch bei dem in \ 328 zitierten jnr $212 \mathrm{mh}$ „2 Steine von 12 Ellen“ wird man wohl „Listenstil“ annehmen dürfen. nomen rectum verleiht dem Referenten der Gesamtverbindung eine besondere Qualität, identifiziert ihn aber nicht eindeutig ${ }^{94}$. In der jüngeren Sprachstufe, nach Ausbildung des Artikelsystems, ist das Rectum in diesem zweiten Fall daher indeterminiert und artikellos, beide Konstruktionen sind formal geschieden und lassen sich auf diese Weise meist deutlich erkennen. Im älteren Ägyptisch kann der Unterschied dagegen nur an der lexikalischen Besetzung des Rectum und aus dem Kontext heraus erkannt werden (z. B. ob z $n \mathrm{~m}^{\top}{ }^{\top} t$,"wahrhaftiger Mann" oder „Mann der [Göttin] Maat" bedeutet).

Wenn man sich die Belege für diese beiden verschiedenen Genetivverbindungen näher ansieht, stellt man fest, daß dieser Unterschied nicht nur darin besteht, ob das nomen rectum spezifisch oder generisch ist, sondern auch (damit zusammenhängend) in der Art der Beziehung zwischen Regens und Rectum liegt. Bezeichnet das Rectum $\left(\mathrm{N}_{2}\right)$ eine spezifische Entität, ist die Relation immer: $\mathrm{N}_{1}$ ist zugehörig $\mathrm{N}_{2}$ bzw. $\mathrm{N}_{1}$ wird $\mathrm{N}_{2}$ zugeordnet (z. B. p3 htm $n$ $p r-3$ „das Siegel des Pharao“ = „das Pharao gehörende Siegel“ oder $n 3$ stj $n t 3$ hrrt ,der Duft der Blu$\mathrm{me}^{\text {" }}=$,der Duft, der zu der Blume $[\mathrm{XY}]$ gehört"). Ist $\mathrm{N}_{2}$ dagegen generisch, lautet die Relation: $\mathrm{N}_{2}$ ist zugehörig $\mathrm{N}_{1}$ bzw. $\mathrm{N}_{2}$ wird $\mathrm{N}_{1}$ zugeordnet ( $p 3$ htm $n$ $n b w$,das Siegel von Gold, das goldene Siegel ${ }^{\text {“ }}=$ „das Siegel, zu dem Gold gehört" oder $n 3$ stj $n$ hrrt „der Blumenduft" $=$ „der Duft, zu dem Blume[naroma] gehört"). Wir haben es also mit einer Art „Richtungsumkehrung“ des Zugehörigkeitsverhältnisses von Regens und Rectum zu tun ${ }^{95}$.

In einem gewissen Sinne ist im ersten Fall $\mathrm{N}_{1}$ das „Objekt" (= das Zugeordnete, das, was von der Zuordnung betroffen ist, das patiens der Zuordnung) und $\mathrm{N}_{2}$ ihr "Subjekt" (= der Fixpunkt des Zuordnungsverhältnisses), im zweiten Fall ist es genau

${ }^{94}$ Mit anderen Worten, der Unterschied zwischen „restringierendem" und „qualifizierendem" Genetiv bestünde nicht zwischen direktem Genetiv einerseits und indirektem andererseits, sondern innerhalb des indirekten Genetivs (und in geringerem Maße auch innerhalb des direkten, s. u.). Allerdings ist es problematisch, im zweiten Fall überhaupt von einem "Genetiv" zu sprechen, s. u., $\int 11$.

${ }^{5}$ Diese Umkehrung ist natürlich etwas ganz anderes als der oben ( $(5$ ) besprochene Unterschied, den H. Junker innerhalb des Genetivs gesehen hat: nomen rectum im Besitz des Regens vs. Regens in abhängiger Beziehung zum Rectum, denn Junkers Unterscheidung beruhte ganz auf der lexikalischen Besetzung und damit auf der Referenz von Regens und Rectum. 
umgekehrt: dort ist $\mathrm{N}_{2}$ in diesem Sinne „Objekt" und $\mathrm{N}_{1}$,Subjekt“. Klarer wird dieses Verhältnis vielleicht an einem (konstruierten) Beispiel: Ein restringierender Genetiv wäre *mdw $n j z$,Wort des Mannes“; ein qualifizierender „Genetiv“ dagegen *z $n j m d w$,Mann des Wortes" (= wortgewandter Mann); und in beiden Fällen wäre $z$ das „Subjekt" und $m d w$ das „Objekt " Tatsächlich sind die Bezeichnungen „Subjekt" und "Objekt" (deren Entsprechung als Aktanten eines transitiven Verbs ja auch eine Art „Richtungsumkehrung“ zugrundeliegt) für ein Genetivverhältnis nicht so weit hergeholt, wie es auf den ersten Blick scheinen mag: „In a number of languages the pronominal affixes that mark the person and number of the possessor are the same as those used for subject function ${ }^{697}$. „The possessive schema is transitive with the possessor encoded as A [ $=$ the agent argument of a transitive verb or any argument that is treated in the same way grammatically] and the possessed as P [ = the patient argument of a transitive verb or any argument that is treated in the same way grammatically] $]^{988}$.

Wenn das so ist, liegt es auf der Hand, wodurch dieser Bedeutungsunterschied erreicht wurde. Denn bei den Nisben gibt es ja eine ganz ähnliche „Richtungsumkehrung“ zwischen „einfachen“ und „umgekehrten“ Nisbeadjektiven: so bedeutet X jmj Y als „einfache" Nisbe „X, der sich in $\mathrm{Y}$ befindet" (d. h. X ist zugehörig Y) und als „umgekehrte" Nisbe „X, in dem sich Y befindet“ (d. h. Y ist zugehörig X). Ähnlich könnte X $n j \mathrm{Y}$ entweder „X, der zu Y gehört" (X zugehörig $\mathrm{Y})$ oder „X, zu dem $\mathrm{Y}$ gehört“ $(\mathrm{Y}$ zugehörig $\mathrm{X}$ ) bedeuten ${ }^{99}$. Es wird also kein Zufall sein, daß wir in älterer Sprache diesen Unterschied ganz auf den indirekten Genetiv beschränkt finden: denn dort werden nomen regens und rectum ja durch ein Nisbeadjektiv verbunden. Wir werden den Genetiv zum „Ausdruck der Zugehörigkeit oder Abhängigkeit des Regens im Verhältnis zum Rectum" also auf einen (indirekten) Genetiv zurückfüh-

\footnotetext{
${ }^{96}$ Ein ähnliches, aber tatsächlich belegtes Paar ist wbnw (nb) $n j z$,(irgend) eine Wunde des Mannes“ (pEbers, 64,13) und $z$ nj wbnw „ein Mann mit einer Wunde" (WGMT, \& 143 [11]).

${ }^{97}$ Blake, Case, 151.

${ }^{98}$ Ibid., 152.

99 Historisch ist diese „Richtungsumkehrung“ auf die Aktiv-Passiv-Diathese zurückzuführen; man könnte die "einfache" Nisbe auch „aktive Nisbe" nennen, die "umgekehrte" passive Nisbe, vgl. Jansen-Winkeln, LingAeg 3, 1993, 7-16.
}

ren, in dem Regens und Rectum durch eine „einfache" (aktive) Nisbe $n j$ verbunden waren, die "qualifizierende Bestimmung des Regens durch das Rectum (Angabe von Maß und Zahl, Stoff, Inhalt, Art und Weise u. ä.)" aber auf die Verbindung durch eine „umgekehrte" (passive) Nisbe.

Allerdings kann dieser Unterschied in der Nisbe $n j$ nur in älterer Zeit (noch) vorhanden gewesen sein. Im Koptischen werden ja "Genetiv" und „Attribut" beide durch $\mathbf{N}$ angeschlossen, und dieser Zusammenfall hat mit Sicherheit schon viel früher stattgefunden. Das kann aber nur heißen, daß sich die Unterscheidung von restringierendem und qualifizierendem Genetiv von der unterschiedlichen Bedeutung (und Bildung) ${ }^{100}$ des verbindenden Nisbeadjektivs auf die unterschiedlichen Determinationsverhältnisse (Rectum des qualifizierenden Genetivs grundsätzlich indeterminiert und artikellos ${ }^{101}$ ) verlagert hat, und die waren erst ab dem Neuägyptischen offen markiert.

Das würde aber zugleich bedeuten, daß im jüngeren Ägyptisch die Unterscheidung von restringierendem und qualifizierendem Genetiv an sich gar nicht mehr auf den indirekten Genetiv beschränkt zu sein brauchte, denn jetzt war sie ja unabhängig von der Art des verbindenden Nisbeadjektivs. Sie wäre jetzt ebensogut im direkten Genetiv denkbar - mit der Einschränkung allèrdings, daß der direkte Genetiv im Neuägyptischen schon viel seltener und weniger produktiv ist als der indirekte. Und tatsächlich finden wir im Neuägyptischen auch eine Reihe derartiger qualifizierender direkter Genetive ${ }^{102}$. Man wird wohl annehmen dürfen, daß nicht alle derartigen Belege nur defektive Schreibungen des indirekten Genetivs $\operatorname{sind}^{103}$. Im älteren Ägyptisch gibt es dagegen erheblich weniger (und viel weniger eindeutige) entsprechende Belege.

${ }^{100} \mathrm{Zu}$ dem formalen Unterschied von „einfachen“ und „umgekehrten“ Nisben s. Osing, Nominalbildung, 309-10.

${ }^{01}$ Vgl. A.Shisha-Halevy, Coptic Grammatical Categories, AnOr 53, 1986, 131 (mit n. 10): „the generic reference effected by the zero article for the second noun determines the qualificative relations between it and the first."

Vgl. Erman, NG, \ 202 und Junge, Einführung in die Grammatik des Neuägyptischen, 63-4.

${ }^{103}$ Übrigens findet sich auch im Spätmittelägyptischen eine Reihe von qualifizierenden direkten Genetiven, vgl. SpmG, \399, zweifellos aufgrund von Beeinflussung durch die gesprochene Sprache. 
11. Die Trennung, die wir im Neuägyptischen zwischen „restringierendem" und „qualifizierendem" Genetiv finden bzw. im Demotischen und Koptischen zwischen „possessiver" und ,attributiver" Beifügung, läßt sich also durch die gesamte ägyptische Sprachgeschichte verfolgen. Der Unterschied ist funktional weitgehend derselbe, nur wird er im älteren Ägyptisch anders markiert als im jüngeren. Allerdings ist die „qualifizierende“ Konstruktion in jüngerer Sprache sehr viel häufiger, weil sie jetzt zusätzlich die attributiven Funktionen der allmählich verschwindenden Kategorie „Adjektiv“ übernimmt.

Fraglich ist allerdings, und zwar für die gesamte Sprachgeschichte, ob man die Konstruktion, die oben als "qualifizierender Genetiv" bezeichnet wurde, überhaupt, selbst cum grano salis, als Genetiv betrachten darf. Vermutlich sollte man es nicht: der Kernbereich dessen, was sonst den funktionalen Hauptaspekt eines „Genetivs" ausmacht (und insofern eine solche Bezeichnung rechtfertigt, s. o., \1), nämlich der Ausdruck des Possessivverhältnisses, wird ja gerade nicht von ihr abgedeckt. Angemessener wäre vielleicht so etwas wie „qualifizierende Beifügung“" o. ä. ${ }^{104}$ Im älteren Ägyptisch ergäbe sich allerdings die praktische Schwierigkeit, daß beide Konstruktionen bei weitem nicht in allen Fällen sicher voneinander zu unterscheiden sind.

12. Das Ägyptische hat zwei unterschiedliche Genetivkonstruktionen, eine unmittelbare Verbindung von nomen regens (zumindest $z$. T. als status constructus gebildet) und nomen rectum und eine „indirekte“ bzw. periphrastische.

Beide Konstruktionen sind zunächst syntaktisch andersartig und verhalten sich dementsprechend auch unterschiedlich: die periphrastische Verbindung ist lockerer und kann leicht getrennt werden. Der indirekte Genetiv kann daher zur Variation und Auflockerung von Wortstellung und Satzbau dienen; es ergeben sich dadurch zweifellos auch stilistische Unterschiede (die uns allerdings großenteils nicht zugänglich sind). Weiter können die beiden Genetivarten der semantischen Nuancierung dienen: der direkte Genetiv drückt wohl eine engere Verbindung von Regens und Rectum aus (z. B. werden einheitliche Begriffe eher durch den direkten Genetiv wiedergegeben), der indirekte Genetiv als (ursprünglich)

${ }^{104}$ Rein formal handelt es sich in älterer Sprache ja ohnehin in beiden Fällen um Attribution eines Nisbeadjektivs. periphrastische Form eine weitere. Oft scheinen allerdings auch beide Konstruktionen mehr oder weniger freie Varianten zu sein (s. o., $\int 7$, Ende). Schließlich gibt es aber auch eine Reihe von Lexemen und Wortformen, wo offenbar keine Wahlmöglichkeit gegeben war: Bei ihnen stehen die beiden Genetivkonstruktionen in komplementärer Distribution (s. o., \8). Und in diesen Fällen kann natürlich kein semantischer oder stilistischer Unterschied zwischen ihnen bestanden haben.

Insgesamt gesehen können die Unterschiede zwischen direktem und indirektem Genetiv nicht auf ein einziges Prinzip zurückgeführt werden. Beide ergänzen sich in verschiedener Hinsicht. Im Verlauf der Sprachgeschichte mit ihrer generellen Tendenz zur Analyse wird der direkte Genetiv als produktive Wortverbindung allmählich seltener; viele direkte Verbindungen erstarren und werden univerbiert, und schließlich bleibt nur noch der indirekte als frei verwendbare Konstruktion übrig.

Beim indirekten Genetiv erlaubt die Art seiner Bildung zwei verschiedene Bedeutungen: je nachdem, ob das verbindende Nisbeadjektiv $n j$ eine „einfache“ oder „umgekehrte“ Nisbe ist, ergeben sich zwei verschiedene „Richtungen“ der Zugehörigkeit von Regens und Rectum: das Regens ist dem Rectum zugehörig oder umgekehrt. Nach der Erstarrung des Nisbeadjektivs zu einer unveränderlichen Partikel wird dieser selbe Unterschied durch die unterschiedlichen (expliziten) Determinationsverhältnisse, die sich inzwischen entwickelt haben, ausgedrückt, und damit wird dieser Unterschied auch beim direkten Genetiv (soweit er noch produktiv ist) möglich. Allerdings sind beide Konstruktionen funktional so unterschiedlich, daß man kaum beide als „Genetiv“ klassifizieren kann.

\section{SUMMARY}

In Egyptian, two different grammatical constructions are classified as "genitive": the so-called "direct" and "indirect genitive". Generally, the two constructions differ in syntax and meaning, but they may occur in free variation as well as in complementary distribution. The differences between them can not be explained by a single principle, they are complementary in many respects. In Later Egyptian, there seem to be two different uses of the indirect genitive: as an expression of belonging or dependence vs. an expression of qualification. It can be shown that these uses already existed in Earlier Egyptian, when they were rendered by two different kinds of nisbeadjectives: the "normal" nisbe to express the relationship of belonging or dependence, and the "reciprocal" nisbe to express qualification. 